\title{
Hyperbolic Orbifolds of Minimal Volume
}

\author{
Ruth Kellerhals
}

\begin{abstract}
We provide a survey of hyperbolic orbifolds of minimal volume, starting with the results of Siegel in two dimensions and with the contributions of Gehring, Martin and others in three dimensions. For higher dimensions, we summarise some of the most important results, due to Belolipetsky, Emery and Hild, by discussing related features such as hyperbolic Coxeter groups, arithmeticity and consequences of Prasad's volume, as well as canonical cusps, crystallography and packing densities. We also present some new results about volume minimisers in dimensions six and eight related to Bugaenko's cocompact arithmetic Coxeter groups.
\end{abstract}

Keywords Hyperbolic orbifold · Minimal volume · Coxeter group · Arithmetic group

Mathematics Subject Classification (2000) $\quad 57 \mathrm{M} 60 \cdot 57 \mathrm{R} 18 \cdot 51 \mathrm{~F} 15 \cdot 22 \mathrm{E} 40$

\section{Introduction}

Let $n \geq 2$, and consider a hyperbolic orbifold $Q$ of dimension $n$, that is, $Q$ is the quotient of $\mathbb{H}^{n}$ by a discrete group of hyperbolic isometries. By a theorem of Kazhdan and Margulis, it is known that the volume of $Q$ is bounded from below by a universal constant $v_{n}>0$. A natural problem is to determine $v_{n}$ and to find orbifolds $Q_{*}$ such that $\operatorname{vol}_{n}\left(Q_{*}\right)=v_{n}$. This problem splits up in a natural way with respect to compactness, orientability, arithmeticity and dimension parity.

R. Kellerhals $(\varangle)$

Department of Mathematics, University of Fribourg, 1700 Fribourg, Switzerland

e-mail: ruth.kellerhals@unifr.ch 
For $n=2$, the above problem was solved by Siegel [27] who showed that the arithmetic Coxeter triangle group [3,7], defined over the field $\mathbb{Q}[\cos (2 \pi / 7)]$ (resp. $[3, \infty]$, defined over $\mathbb{Q}$ ) provides the unique compact (resp. non-compact) hyperbolic two-orbifold of minimal area $\pi / 42$ (resp. $\pi / 6$ ). Siegel's proof is based on a careful analysis of his formula for the Euler characteristic.

For $n=3$, the minimal volume problem has a longer history of development to full solution. In the arithmetic case, Chinburg and Friedman [6] proved that the Coxeter tetrahedral group $[3,5,3]$ provides, in a unique way, the smallest representative in the class of orientable arithmetic three-orbifolds. The group $[3,5,3]$ is defined over the field $k_{1}=\mathbb{Q}\left[(3+2 \sqrt{5})^{1 / 2}\right]$ with discriminant -275 . By Borel's formula, its covolume is given by

$$
\operatorname{vol}_{3}\left(\mathbb{H}^{3} /[3,5,3]\right)=\frac{275^{3 / 2}}{8 \pi^{2}} \zeta_{k_{1}}(2) \simeq 0.03905 .
$$

In the non-compact case, Meyerhoff [25] proved that the singly cusped quotient of $\mathbb{H}^{3}$ by the orientation preserving subgroup of the Coxeter simplex $[3,3,6]$ is of minimal volume among all oriented non-compact hyperbolic 3 -orbifolds. This orbifold can also be represented as quotient space by the arithmetic group $\operatorname{PSL}\left(2, \mathcal{O}_{3}\right)$, where $\mathcal{O}_{3}$ is the ring of integers of the field $k_{2}=\mathbb{Q}[\sqrt{-3}]$. The volume can be computed, first by considering an ideal regular hyperbolic tetrahedron and by using Milnor's formula [26, Lem. 2], and secondly by Humbert's volume formula (corrected by Grunewald and Kühnlein [15]) applied to $P S L\left(2, \mathcal{O}_{3}\right)$. In this way, one obtains the interesting identity

$$
\operatorname{vol}_{3}\left(\mathbb{H}^{3} / \operatorname{PSL}\left(2, \mathcal{O}_{3}\right)\right)=\frac{1}{4} \mathrm{JI}\left(\frac{\pi}{3}\right)=\frac{3^{3 / 2}}{8 \pi^{2}} \zeta_{k_{2}}(2) \simeq 0.08456
$$

where $\mathrm{JI}(x)$ is the Lobachevsky function [see (2.8)]. The proof of Meyerhoff is based on several ingredients, ranging from Jørgensen's trace inequality for discrete twogenerator subgroups in $P S L(2, \mathbb{C})$, Euclidean crystallography and wall-paper groups to (horo-) sphere packings and Böröczky's local density bound.

Recently, in a series of articles, Martin together with Gehring [13,14], Marshall [24] and with Maclachlan and Reid [12], settled the minimal volume problem in a complete and unified way. The works of Martin and his coauthors, put together, prove that the space $\mathbb{H}^{3} /[3,5,3]$ is the unique three-orbifold of minimal volume. The proof consists of several different parts, beginning with the fact that the rotation subgroup of $[3,5,3]$ is a two-generator, discrete, non-elementary subgroup of $\operatorname{PSL}(2, \mathbb{C})$ of restricted order elliptics.

In this work, we shall give a survey of hyperbolic $n$-orbifolds of minimal volume for dimensions $\geq 4$. We present some of the most important results, in particular those due to Belolipetsky, Emery and Hild, and we discuss related concepts such as hyperbolic Coxeter groups, arithmeticity and the impact of Prasad's volume, as well as canonical cusps, crystallography and packing densities. Our focus will be on the geometriccombinatorial side. For the arithmetic side, with its involved techniques, we refer to $[3,7]$ and the bibliographies therein. Finally, we present some new results by identifying orientable compact arithmetic volume minimisers in dimensions six and eight with the quotient spaces of the correspondent Coxeter groups discovered by Bugaenko. 


\section{Candidates for Minimal Volume}

Promising candidates for hyperbolic orbifolds of minimal volume are orbit spaces by discrete groups of hyperbolic isometries with simplest presentations, that is, with a set of few generators related by simple relations. Beside this algebraic-combinatorial aspect, minimal volume realisations seem to be arithmetic, at least in low dimensions. Since each hyperbolic isometry is a product of finitely many reflections in hyperplanes of hyperbolic space, it is natural to look at discrete hyperbolic reflection groups, their fundamental polytopes and arithmetic examples.

\subsection{Hyperbolic Coxeter Groups with Few Generators}

Denote by $\mathbb{X}^{n}, n \geq 2$, either the hyperbolic space $\mathbb{H}^{n}$, the unit sphere $\mathbb{S}^{n}$, or the Euclidean space $\mathbb{E}^{n}$, and let $\mathbb{H}^{n}$ be embedded in the Lorentz-Minkowski vector space $\mathbb{E}^{n, 1}$ of signature $(n, 1)$. A geometric Coxeter group $G=(W, S) \subset \operatorname{Isom}\left(\mathbb{X}^{n}\right)$ is a discrete group $W$ generated by finitely many reflections $s \in S$ in hyperplanes $H_{S}$ of $\mathbb{X}^{n}$ subject to $s^{2}=1$ and to relations of the form

$$
\left(s s^{\prime}\right)^{m}=1 \text { for an integer } m=m\left(s, s^{\prime}\right) \geq 2,
$$

for distinct generators $s, s^{\prime} \in S$ with mirrors $H_{S}, H_{S^{\prime}}$ intersecting in $\mathbb{X}^{n}$. A fundamental polytope for $G$ is called a Coxeter polytope $P_{S} \subset \mathbb{X}^{n}$. It arises as a convex polytope bounded by an isometric set of images of the mirrors $H_{s}, s \in S$, which either intersect in $\mathbb{X}^{n}$ under the dihedral angle $L\left(H_{s}, H_{s^{\prime}}\right)=\pi / m, s \neq s^{\prime}$, or-if there is no relation as in (2.1) - they are parallel if $\mathbb{X} \neq \mathbb{S}^{n}$, or admit a common perpendicular, in the case $\mathbb{X}^{n}=\mathbb{H}^{n}$ only. We are particularly interested in hyperbolic Coxeter $n$-polytopes of small volume. Such polytopes and Coxeter groups are related to small cardinality $|S|$. For $|S|=n+l, l \leq 3$, there is a complete classification (cf. [32, Part II, Cha. 5] and [29]). In particular, for $l=1$, the list of compact Coxeter simplices is due to Lannér and contains examples up to dimension 4 while the list of non-compact finite volume Coxeter simplices, sometimes termed quasi-Lannér simplices, was established by Koszul and contains examples up to dimension 9 (cf. [32, Part II, Cha. 5, Sec. 2.3]). For $l=2$, Coxeter polytopes of finite volume are combinatorially either products of two simplices or pyramids over a product of simplices. This fact is the background for their classification performed by Kaplinskaja, Esselmann and Tumarkin (cf. [28], for example). The corresponding list ends in dimension 17 where the non-compact Coxeter pyramid $P_{*}$ (see Fig. 1), discovered by Vinberg [31, p. 65], is intimately related to the automorphism group $P O\left(\mathrm{II}_{17,1}\right)$ of the even unimodular Lorentzian lattice $\mathrm{II}_{17,1}$ in $\mathbb{R}^{18}$ (see also 4.3.1).

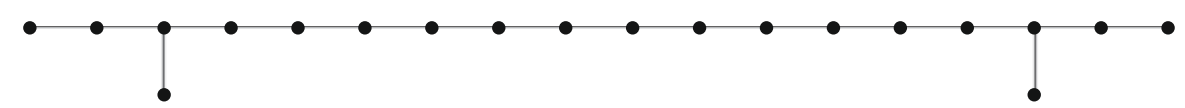

Fig. 1 The Coxeter pyramid $P_{*}$ in $\mathbb{H}^{17}$ 
Coxeter polytopes $P_{S}$ and Coxeter groups $G=(W, S)$ with small $|S|$ are most conveniently described by their Coxeter graph $\Sigma$. To each bounding hyperplane $H_{S}$ indexed by a generator $s \in S$ corresponds a node $v_{S}$ of the graph $\Sigma$. An edge with weight $m$ is drawn between two nodes $v_{s}, v_{S^{\prime}}$ in $\Sigma$ if the associated hyperplanes $H_{S}, H_{S^{\prime}}$ intersect under the angle $\pi / m$ for $m \geq 3$ [see (2.1)]. For $m=3$, an instance arising very frequently, the edge carries no weight. If $H_{s}, H_{s^{\prime}}$ are perpendicular (parallel, or admitting a common perpendicular), the nodes $v_{s}, v_{s^{\prime}}$ are not joined (or joined by an edge with weight $\infty$, or joined by a dotted edge). In order to encode the shape of a Coxeter graph in an abbreviated way we often use the Coxeter symbol. For example, $[p, q, r]$ is associated to a linear Coxeter graph with 3 edges of consecutive markings $p, q, r$. The Coxeter symbol $\left[3^{i, j, k}\right]$ denotes a group with Y-shaped Coxeter graph with strings of $i, j$ and $k$ edges emanating from a common node. Notice that dotted edges are not encoded. Consider the following examples. The Coxeter graphs $\Sigma_{k}$

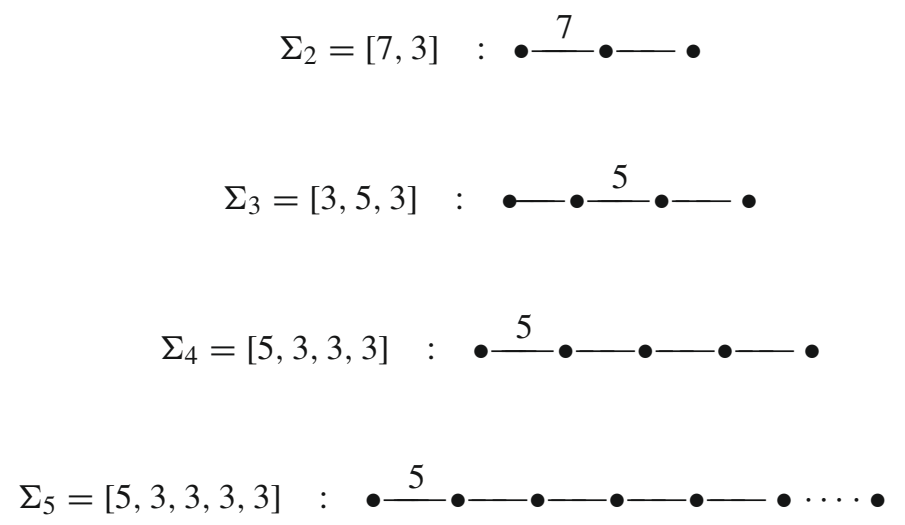

describe arithmetic compact Coxeter orthoschemes (described by linear graphs) in $\mathbb{H}^{k}$ for $k=2,3,4$, while $\Sigma_{5}$ describes an arithmetic compact straight simplicial Coxeter 5 -prism with basis given by the hyperbolic 4-Coxeter simplex with graph $\Sigma_{4}$. Of particular interest is the hyperbolic arithmetic Coxeter group $\Sigma_{*}=\left[3^{2,1}, 3^{13}, 3^{2,1}\right]$ with 19 generators and Coxeter graph given by Fig. 1. A fundamental domain is the non-compact Coxeter pyramid $P_{*} \subset \mathbb{H}^{17}$ with apex $q \in \partial \mathbb{H}^{17}$ over a product of two (Euclidean) Coxeter simplices (of type $\widetilde{E_{8}}$ ). In this context, notice that Tumarkin [28] classified all non-compact, finite volume Coxeter pyramids and proved that they exist in $\mathbb{H}^{n}$ for $n \leq 17$, only.

Finally, let us point out that efficient arithmeticity criteria for hyperbolic Coxeter groups were described by Vinberg and are comparatively easy to apply (cf. [32, Part II, Cha. 6, Sec. 3.1], for example).

\subsection{Volumes of some hyperbolic Coxeter polytopes}

The volumes of all hyperbolic Coxeter simplices (existing in $\mathbb{H}^{n}$ for $n \leq 9$ ) have been calculated in [18] by the application of several methods, ranging from analytical, combinatorial to arithmetic techniques. In Table 1, we present the minimal (and some 
Table 1 Some small volume hyperbolic Coxeter $n$-simplices

\begin{tabular}{llll}
\hline Dim $n$ & Coxeter symbol & Compact, arithmetic & Volume \\
\hline 3 & {$[4,3,5]$} & Yes, Yes & $\simeq 0.03588$ \\
3 & {$[3,5,3]$} & Yes, Yes & $\simeq 0.03905$ \\
3 & {$[3,3,6]$} & No, Yes & $\simeq 0.04229$ \\
4 & {$[3,3,3,5]$} & Yes, Yes & $\frac{\pi^{2}}{10,800} \simeq 0.00091$ \\
4 & {$\left[4,3^{2,1}\right]$} & No, Yes & $\frac{\pi^{2}}{1,440} \simeq 0.00685$ \\
5 & {$[3,3,3,4,3]$} & No, Yes & $\frac{7 \zeta(3)}{40,800} \simeq 0.00018$ \\
5 & {$\left[4,3,3^{2,1}\right]$} & No, Yes & $\frac{7 \zeta(3)}{15,360} \simeq 0.00055$ \\
\hline
\end{tabular}

small) volume hyperbolic Coxeter $n$-simplices, for $n \leq 5$, by distinguishing with respect to compactness and arithmeticity. Observe that, in the list, hyperbolic Coxeter polytopes with many dihedral angles of type $\pi / 2$ and $\pi / 3$ occur. This is compatible with the volume growth with respect to infinitesimal dihedral angle decay as given by Schläfli's volume differential formula (see [32, Part I, Cha. 7, Sec. 2.2], for example). For hyperbolic $n$-simplices $S$, this formula says that

$$
d \operatorname{vol}_{n}(S)=\frac{-1}{n-1} \sum_{F \subset S} \operatorname{vol}_{n-2}(F) d \alpha_{F},
$$

where $F$ ranges over all codimension 2 faces of $S$, with attached dihedral angle $\alpha_{F}$, and where $\operatorname{vol}_{0}:=1$. Observe that the volume of the compact Coxeter tetrahedron $[3,5,3]$ is larger than the volume of $[4,3,5]$. However, the symbol $[3,5,3]$ and its graph $\Sigma_{3}$ have an internal symmetry indicating that the tetrahedron [3, 5, 3] admits a symmetry plane $H$ dissecting it into two isometric copies of half of its volume. By extending the Coxeter group $G=[3,5,3]$ by the group generated by the corresponding half-turn $\gamma_{H} \in \operatorname{Isom}\left(\mathbb{H}^{3}\right)$, one obtains a new discrete group $G^{\prime} \subset \operatorname{Isom}\left(\mathbb{H}^{3}\right)$ of smaller volume than $[4,3,5]$. Notice that $G^{\prime}$ is not a Coxeter group anymore.

Consider the cocompact arithmetic Coxeter group associated to the Coxeter 5-prism with graph given by $\Sigma_{5}$ [see (2.2d)]. Its covolume has been determined in [10, (5.1)], in a twofold way. One approach is based on Schläfli's formula (2.3), suitably adapted for hyperbolic five-polytopes. It allows us to express $\operatorname{vol}_{5}\left(\Sigma_{5}\right)$ as a sum of simple integrals over dilogarithmic functions as follows (see [19] and [22, Sec. 4.2]).

$$
\operatorname{vol}_{5}\left(\Sigma_{5}\right)=\frac{1}{4} \int_{\pi / 3}^{2 \pi / 5} F(t) d t+\frac{\zeta(3)}{3200} \simeq 0.00076729618
$$

where the integrand $F(t)=\operatorname{vol}_{3}([5,3, \beta(t)])$ is the volume of the hyperbolic orthoscheme $[5,3, \beta(t)]$ ) with (non-right) dihedral angles $\pi / 5, \pi / 3, \beta(t)$ where

$$
\left.\beta(t)=\arctan \sqrt{2-\cot ^{2} t} \in\right] 0, \frac{\pi}{2}[.
$$


Put

$$
\left.\theta(t)=\arctan \frac{\sqrt{1-4 \sin ^{2} \frac{\pi}{5} \sin ^{2} \beta(t)}}{2 \cos \frac{\pi}{5} \cos \beta(t)} \in\right] 0, \frac{\pi}{2}[.
$$

Then, $F(t)=\operatorname{vol}_{3}([5,3, \beta(t)])$ is given by Lobachevsky's formula (see [32, Part I, Cha. 7, Sec. 3.3], for example) according to

$$
\begin{aligned}
F(t)= & \frac{1}{4}\left\{\mathrm{JI}\left(\frac{\pi}{5}+\theta(t)\right)-\mathrm{JI}\left(\frac{\pi}{5}-\theta(t)\right)-\mathrm{JI}\left(\frac{\pi}{6}+\theta(t)\right)+\right. \\
& \left.+\mathrm{JI}\left(\frac{\pi}{6}-\theta(t)\right)+\mathrm{J}(\beta(t)+\theta(t))-\mathrm{J}(\beta(t)-\theta(t))+2 \mathrm{JI}\left(\frac{\pi}{2}-\theta(t)\right)\right\} .
\end{aligned}
$$

Here,

$$
\mathrm{JI}(\omega)=\frac{1}{2} \sum_{r=1}^{\infty} \frac{\sin (2 r \omega)}{r^{2}}=-\int_{0}^{\omega} \log |2 \sin t| d t, \omega \in \mathbb{R}
$$

is Lobachevsky's function. For the second approach, we exploit the fact that the Coxeter group $\Sigma_{5}$ is arithmetic with defining field $k_{0}=\mathbb{Q}(\sqrt{5})$ so that we can use the number theoretical machinery around Prasad's volume formula. In Section 4.2.1, we explain how to derive by arithmetic means the interesting identity [see (2.4-2.8)]

$$
\operatorname{vol}_{5}\left(\Sigma_{5}\right)=\frac{1}{4} \int_{\pi / 3}^{2 \pi / 5} F(t) d t+\frac{\zeta(3)}{3200}=\frac{9 \sqrt{5}^{15}}{2^{15} \pi^{15}} \zeta_{k_{0}}(2) \zeta_{k_{0}}(4) L_{\ell_{0} / k_{0}}(3) \simeq 0.00076
$$

where the fields involved are $k_{0}$ and $\ell_{0} \cong \mathbb{Q}[x] /\left(x^{4}-x^{3}+3 x-1\right)$. Here, the symbol $\zeta_{k}$ denotes the Dedekind zeta function associated with $k$, and $L_{\ell / k}=\zeta_{\ell} / \zeta_{k}$ is the $L$-function corresponding to a quadratic extension $\ell / k$.

For the computation of some of the hyperbolic Coxeter pyramids, we refer to $[11,19$, 20]. For the (single) top-dimensional Coxeter pyramid $P_{*} \subset \mathbb{H}^{17}$, which is, moreover, non-compact, with Coxeter symbol $\Sigma_{*}=\left[3^{2,1}, 3^{13}, 3^{2,1}\right]$ and graph according to Fig. 1, Emery [8] determined recently its volume by arithmetic tools (see also 4.3.1). He obtained

$$
\operatorname{vol}_{17}\left(P_{*}\right)=\frac{691 \times 2617}{2^{38} \times 3^{10} \times 5^{4} \times 7^{2} \times 11 \times 13 \times 17} \zeta(9) \simeq 2.072451981 \times 10^{-18} .
$$

\section{Minimal Volume in the Presence of Cusps}

Consider a non-compact $n$-orbifold $Q=\mathbb{H}^{n} / \Gamma$ of finite volume. Then, $\Gamma$ contains at least one parabolic isometry $\gamma$, and a fundamental polyhedron $P \subset \mathbb{H}^{n}$ for $\Gamma$ has 
at least one ideal vertex $q \in \partial \mathbb{H}^{n}$ stabilised by the subgroup $\Gamma_{q} \subset \Gamma$ generated by the parabolic element $\gamma$. The subgroup $\Gamma_{q}$ is a crystallographic group of Euclidean isometries containing, by results of Bieberbach, a translational lattice $\Lambda$ of rank $n-1$. We identify $\Lambda$ with a full rank $\mathbb{Z}$-module of vectors in $\mathbb{E}^{n-1}$. By passing to the upper half space model for $\mathbb{H}^{n}$ in $U^{n}=\left(\mathbb{E}_{+}^{n}, d s^{2}=\left(\mathrm{d} x_{1}^{2}+\cdots+\mathrm{d} x_{n-1}^{2}+d t^{2}\right) / t^{2}\right)$, we can identify, without loss of generality, $q=\infty$ and analyse the actions of $\Gamma_{\infty}$ and $\Lambda_{\infty}$ on each (Euclidean) horosphere $S_{h}=\left\{(x, t) \in \mathbb{E}_{+}^{n} \mid t=h\right\}, h>0$, based at infinity and bounding the horoball $B_{h}=\left\{(x, t) \in \mathbb{E}_{+}^{n} \mid t>h\right\}$.

Inspired by the approach of Meyerhoff, Kellerhals [21], Hild-Kellerhals [17] and Hild [16] extended these ideas to and developed suitable methods for arbitrary dimensions, and this in the orientation free context. In [16,17], respectively, they applied these methods for $n=4$ and for $5 \leq n \leq 9$, respectively, in order to derive the following volume minimality results, and by retrieving the results of Siegel for $n=2$ and of Meyerhoff for $n=3$ (see Sect. 1).

Theorem 1 For $4 \leq n \leq 9$, let $Q_{n}=\mathbb{H}^{n} / \Gamma_{n}$ be a cusped hyperbolic $n$-orbifold of minimal volume. Then, up to isomorphism, $\Gamma_{n}$ is the hyperbolic Coxeter simplex group according to Table 2, and as such it is uniquely determined.

The values in Table 2 are expressed in terms of the Riemann zeta function $\zeta$, the Lobachevsky function JI (see [2.8)] and a Dirichlet $L$-series (see [18]).

In what follows, we summarise the methods and sketch the proof (for details and references, see [16]). Start from a finite volume hyperbolic $n$-orbifold $Q=\mathbb{H}^{n} / \Gamma$ such that $\Gamma_{\infty} \neq 1$ as above and consider the projection $\eta: \Gamma_{\infty} \rightarrow O(n-1)$ which sends a Euclidean isometry $x \mapsto A x+a$ to $A$. The image $\Phi_{\infty}=\operatorname{im}(\eta)$, called finite point group, is a subgroup of $\Gamma_{\infty}$, and $i_{\infty}:=\left[\Gamma_{\infty}: \Lambda_{\infty}\right]=\left|\Phi_{\infty}\right|$, if and only if $\Gamma_{\infty}$ is symmorphic, that is, the $\Gamma_{\infty}$-orbit of $0 \in \mathbb{E}^{n-1}$ equals the translational lattice $\Lambda_{\infty}$. Denote by $D \subset \mathbb{E}^{n-1}$ a fundamental polytope for $\Lambda_{\infty}$, and suppose without loss of generality that the minimal translation length of vectors in $\Lambda_{\infty}$ equals 1 . For the associated horoball $B_{\infty}:=B_{1} \subset U^{n}$, the $\Gamma$-images of $B_{\infty}$ (and their orthogonal projections to $\left\{x_{n}=0\right\}$ ) provide a horoball packing of $U^{n}$ (and a sphere packing of $\mathbb{E}^{n-1}$ ) where the upper bound $d_{n}(\infty)$ for local densities (and classical maximal density results up to dimension $n-1=8$ ) is at our disposal. Furthermore, it is known that $B_{\infty}$ projects to an embedded cusp neighborhood $C_{\infty} \subset Q$, and that $C_{\infty}$ is disjoint from all cusp neighborhoods arising from different, non-conjugate parabolic subgroups in $\Gamma$. Due to this fact, each of these cusp neighborhoods is called a canonical cusp and denoted by $C_{\text {can }}$. They form the set $\mathcal{C}$ of canonical cusps of $Q$. These observations lead to the following volume relations. For our preferred canonical cusp $C_{\text {can }}=B_{\infty} / \Gamma_{\infty}$, we derive the expression

$$
\operatorname{vol}_{n}\left(C_{\mathrm{can}}\right)=\frac{\operatorname{vol}_{n-1}(D)}{(n-1) i_{\infty}}
$$

while globally,

$$
\operatorname{vol}_{n}(Q) \geq \frac{1}{d_{n}(\infty)} \sum_{C_{\mathrm{can}} \in \mathcal{C}} \operatorname{vol}_{n}\left(C_{\mathrm{can}}\right)
$$


Table 2 Minimal volume cusped hyperbolic $n$-orbifolds

Dim $n$
$\frac{1}{6} \simeq 0.5236$
$\frac{1}{8} \pi\left(\frac{\pi}{3}\right) \simeq 0.0423$
$\frac{\pi^{2}}{1,440} \simeq 6.85 \times 10^{-3}$
$\frac{7 \zeta(3)}{46,080} \simeq 1.82 \times 10^{-4}$
$\frac{\pi^{3}}{777,600} \simeq 3.98 \times 10^{-6}$
$\frac{\sqrt{3} L(4,3)}{1,720,320} \simeq 9.46 \times 10^{-7}$
7

Therefore, the volume bound depends on the crystallographic data such as $D$ and $i_{\infty}$ on each canonical cusp boundary.

Crystallographic groups acting on $\mathbb{E}^{k}$ are completely classified up to dimension $k=$ 4 by Brown, Bülow, Neubüser, Wondratschek and Zassenhaus. For $5 \leq k \leq 8$, they were studied intensively by Plesken and Pohst who determined all $\mathbb{Z}$ - and $\mathbb{Q}$-classes of maximal, finite, absolutely irreducible subgroups of $G L(k, \mathbb{Z})$. In this context, let $\varphi_{k}$ be the maximal point group order of elements in a fixed $\mathbb{Q}$-class in dimension $k$. The identification of the associated inequivalent lattices together with their fundamental domains is then possible and shows that there is a single lattice of minimal volume $v_{k}$ underlying the densest Euclidean lattice packing in $\mathbb{E}^{k}$. The symmorphic group $S_{k}$ corresponding to its $\mathbb{Z}$-class is related to a Euclidean Coxeter simplex group $\Delta_{k}$. With this background, declare a cusped hyperbolic $n$-orbifold $Q=\mathbb{H}^{n} / \Gamma, n \leq 9$, to be small if

$$
\operatorname{vol}_{n}\left(Q_{n}\right)<2 \frac{v_{n-1}}{(n-1) d_{n}(\infty) \varphi_{n-1}} .
$$

Then, one can show that a small cusped $n$-orbifold has only one cusp and that fullsized horoballs (images of $B_{\infty}$ ) are permuted by the lattice $\Lambda_{\infty}$. For a minimal volume orbifold $Q_{n}=\mathbb{H}^{n} / \Gamma_{n}$, these facts allow one to identify the (unique up to conjugacy) crystallographic group in $\Gamma_{n}$ with an isomorphic copy of $S_{n-1}$, and to show that the associated canonical cusp $C_{\text {can }}$ is maximal, that is, inflating the canonical horoball $B_{\infty}$ covering $C_{\text {can }}$ leads to bigger horoballs whose projections to $Q_{n}$ fail to be embedded. 
This last property implies that the reflection $\sigma$ with respect to the bisector $H_{0}$ of $B_{\infty}$ and its image $B_{0}$ based at $0 \in \mathbb{E}^{n}$ is an element of the group $\Gamma_{n}$. A crucial step is the determination of the combinatorial structure of the Ford domain $P_{F}$ of $\Gamma_{n}$. To this end, observe that $P_{F}$ is the intersection of the Dirichlet-Voronor cell of $B_{\infty}$ with the straight cylinder $Z_{n}=\Delta_{n-1} \times(0, \infty)$ over the Euclidean Coxeter simplex $\Delta_{n-1}$. Then, one proves - by a case-by-case analysis with respect to $n$ - that $P_{F}$ is the simplex of finite volume with vertex at $\infty$ arising from $Z_{n}$ by a cut along the bisector $H_{0}$. The construction of $P_{F}$ reveals that the reflection in each boundary hyperplane of $P_{F}$ belongs to $\Gamma_{n}$ and that the intersection angle of $H_{0}$ with the hyperplane $H_{1}$ bisecting $B_{\infty}$ and one full-sized horoball touching $B_{\infty}$ equals $\pi / 3$. As a result, the group $\Gamma_{n}$ can be identified with the hyperbolic Coxeter simplex group arising from the Euclidean Coxeter graph $\Delta_{n-1}$ by adding one node for $H_{0}$ together with an edge (without weight).

\section{Minimal volume of arithmetic quotients}

Consider an orientable hyperbolic $n$-orbifold $Q=\mathbb{H}^{n} / \Gamma$ of dimension $n \geq 4$ and suppose that the discrete subgroup $\Gamma \subset \operatorname{Isom}^{+}\left(\mathbb{H}^{n}\right)$ is arithmetic with defining field $k$. For the definition of arithmeticity, we refer to [3, Sec. 2] and [7].

\subsection{The even-dimensional case}

For even dimensions $n=2 r \geq 4$, and by distinguishing between the compact case and the non-compact case, Belolipetsky [1,2] showed that there is a corresponding unique orientable arithmetic hyperbolic $n$-orbifold $O=\mathbb{H}^{n} / \Gamma$ of minimal volume. In the compact case, he proved the remarkable fact that the defining field is always equal to $k_{0}=\mathbb{Q}[\sqrt{5}]$. Furthermore, by exploiting Prasad's fundamental volume formula for arithmetic lattices and Bruhat-Tits theory, he determined the explicit values for their Euler characteristic $\chi(O)$ which, by the theorem of Gauss-Bonnet, are related to volume according to

$$
\operatorname{vol}_{n}(O)=\frac{(2 \pi)^{r}}{1 \times 3 \times \cdots \times(2 r-1)} \times|\chi(O)|
$$

The uniqueness aspect is not trivial and based on delicate Galois cohomological arguments. For $n=4$, it allowed him to identify the minimal volume compact orbifold $O_{\mathrm{c}}$ with the oriented double cover of the Coxeter orbifold $\mathbb{H}^{4} /[5,3,3,3]$ since (compare [1, Tab. 2] and Table 1)

$$
\operatorname{vol}_{4}\left(O_{c}\right)=\frac{4}{3} \chi\left(O_{c}\right)=\frac{1}{5,400}=2 \times \operatorname{vol}_{4}([5,3,3,3]) .
$$

Here are the precise statements of the results of Belolipetsky, followed by some explicit $\chi$-values (see [1, Tab. 2]). We start with the compact case. 
Theorem 2 For each dimension $n=2 r \geq 4$ there is a unique orientable compact arithmetic hyperbolic $n$-orbifold $O_{\mathrm{c}}$ of minimal volume. It is defined over the field $k_{0}=\mathbb{Q}[\sqrt{5}]$ and has Euler characteristic

$$
\left|\chi\left(O_{c}\right)\right|=\frac{\lambda(r)}{4^{r-1}} \prod_{i=1}^{r}\left|\zeta_{k_{0}}(1-2 i)\right|
$$

where $\lambda(r)=1$ if $r$ is even, and $\lambda(r)=\left(4^{r}-1\right) / 2$ if $r$ is odd.

In the non-compact case, where the field of definition is always equal to $\mathbb{Q}$, Belolipetsky's result is expressible in terms of Riemann's zeta function as follows.

Theorem 3 For each dimension $n=2 r \geq 4$ there is a unique orientable non-compact arithmetic hyperbolic $n$-orbifold $O_{\mathrm{nc}}$ of minimal volume. It has Euler characteristic

$$
\left|\chi\left(O_{\mathrm{nc}}\right)\right|=\frac{\mu(r)}{2^{r-2}} \prod_{i=1}^{r}|\zeta(1-2 i)|,
$$

where $\mu(r)=1$ if $r \equiv 0,1(\bmod 4)$, and $\mu(r)=\left(2^{r}-1\right) / 2$ if $r \equiv 2,3(\bmod 4)$.

\begin{tabular}{|c||c|c|c|c|c}
\hline$n=2 r \geq 4$ & 4 & 6 & 8 & 10 & $\cdots$ \\
\hline \hline$\left|\chi\left(O_{c}\right)\right|$ & $\frac{1}{7,200}$ & $\frac{67}{576,000}$ & $\frac{24,187}{8,709,120,000}$ & $\frac{309,479,461,547}{3,483,648,000,000}$ & $\cdots$ \\
\hline$\left|\chi\left(O_{\mathrm{nc}}\right)\right|$ & $\frac{1}{960}$ & $\frac{1}{207,360}$ & $\frac{1}{348,364,800}$ & $\frac{1}{91,968,307,200}$ & $\cdots$ \\
\hline
\end{tabular}

\subsubsection{Minimal volume realisation in dimensions six and eight}

In general, it is difficult to construct a compact (arithmetic) hyperbolic orbifold of dimension $n>3$ whose volume is not only computable but also very small. In the even-dimensional cases, volume computation can be reduced to a lower dimensional spherical volume computation which-in the algebraic-topological setting-can be performed by looking at the Euler characteristic.

For $n=6,7$ and 8, Bugaenko [4,5] constructed compact hyperbolic Coxeter groups $B_{n} \subset \operatorname{Isom}\left(\mathbb{H}^{n}\right)$ by arithmetic considerations. More precisely, in [4], he studied the quadratic form $f_{n}$ of signature $(n, 1)$ over the ring $\mathbb{Z}[\omega], \omega=(\sqrt{5}+1) / 2$, given by

$$
f_{n}(x)=x_{1}^{2}+\cdots+x_{n}^{2}-\omega x_{n+1}^{2}
$$

and proved, by applying Vinberg's algorithm, that the automorphism group $O\left(f_{n}\right.$, $\mathbb{Z}[\omega])$ is reflective for $n \leq 7$, only. Furthermore, the associated Coxeter groups $B_{n}$ are all cocompact. The fundamental polytope $P_{B_{6}}$ of $B_{6}$ has 9 facets, and the polytope $P_{B_{7}}$ of $B_{7}$ has 11 facets.

For $n=8$, he constructed in [5, Thm. 3.4] a quadratic form $h$ of signature $(8,1)$ with discriminant $-(1+\sqrt{5})$ over $\mathbb{Z}[\omega]$ for which the group $O(h, \mathbb{Z}[\omega])$ is reflective. 
Fig. 2 The Bugaenko group $B_{6}$

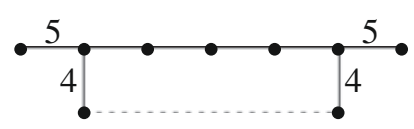

The associated Coxeter group $B_{8}$ is cocompact with fundamental polytope $P_{B_{8}}$ having 11 facets (see Fig. 3).

Bugaenko's polytopes $P_{B_{n}}, 6 \leq n \leq 8$, are highly remarkable. Indeed, in [29], Tumarkin classified all compact Coxeter polytopes in $\mathbb{H}^{n}$ with $n+3$ generators and proved that these polytopes exist only up to dimension $n=8$. Furthermore, for $n=6$ and $n=8$, there is precisely one such $n$-polytope, the Bugaenko polytope $P_{B_{n}}$, while, for $n=7$, there is no compact Coxeter polytope with ten facets at all. In [30], Tumarkin and Felikson studied the related classification problem for compact Coxeter polytopes in $\mathbb{H}^{n}$ with $n+4$ generators. They proved that such polytopes do not exist for $n \geq 8$ and that, for $n=7$, there is precisely one such polytope, and it equals $P_{B_{7}}$.

For $n=6$, the Coxeter graph of Bugaenko's group $B_{6}$ is given by Fig. 2 .

Notice that the Coxeter graph of $B_{6}$ has an internal twofold symmetry, a fact which holds for all Bugaenko groups $B_{n}$ with $n=6,7,8$. Denote by $B_{n}^{\prime}$ the corresponding $\mathbb{Z}_{2}$-extension of the group $B_{n}$.

Proposition 1 The orientable double cover of the orbifold $\mathbb{H}^{6} / B_{6}^{\prime}$ is the unique orientable compact arithmetic 6-orbifold $O_{\mathrm{c}}$ of minimal volume.

Proof We first compute the group Euler characteristic $\chi$ of the cocompact Coxeter group $B_{6}$ with generating set $S$ consisting of 9 reflections by using Steinberg's formula (see $[23,(1.2)]$, for example), that is,

$$
\chi=\sum_{\substack{B_{T} \subset B_{6} \\ \text { finite }}} \frac{(-1)^{|T|}}{\left|B_{T}\right|},
$$

which allows us to express $\chi$ in terms of the orders $\left|B_{T}\right|$ of the finite Coxeter subgroups $B_{T}, T \subset S$, of $B_{6}$. Each such Coxeter subgroup $B_{T} \subset B_{6}$ arises as the stabiliser of a certain face of $P_{B_{6}}$. The orders of their irreducible components have been determined by L. Schläfli (see [19, Sec. 2], for example). Furthermore, the irreducible components given by $\bullet \cdots \bullet$ and by the triangle subgroup $[4,5]$ of $B_{6}$ are hyperbolic and therefore infinite. A careful analysis, and by counting multiplicities of the finite subgroups of $B_{6}$, leads to the following results. First, the $f$-vector of $P_{B_{6}}$ equals $(23,69,95,75,35,9)$ where the components $f_{k}$ are equal to the numbers of $k$-dimensional faces of $P_{B_{6}}$ and satisfy Euler's equation $f_{0}-f_{1}+f_{2}-f_{3}+f_{4}-f_{5}=0$ as well as the simplicity condition $f_{1}=3 f_{0}$. Next, we compute

$$
\chi=-\frac{67}{576,000} .
$$

Finally, cut the polytope $P_{B_{6}}$ along the hyperplane $H$, with associated half-turn $\gamma_{H}$, into two isometric pieces as indicated in 2.2. The isometry $\gamma_{H}$ generates a copy of $\mathbb{Z}_{2}$ 
Fig. 3 The Bugaenko group $B_{8}$

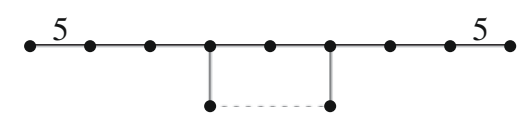

so that each piece is a fundamental domain of the (arithmetic) $\mathbb{Z}_{2}$-extension $B_{6}^{\prime}$ of $B_{6}$. It follows that the oriented double cover $O_{6}$ of $\mathbb{H}^{6} / B_{6}^{\prime}$ has Euler characteristic given by (4.5) which coincides with the minimal one for $n=6$ (see the table following Theorem 3). By the uniqueness statement in Theorem 3, the orbifold $O_{6}$ is isometric $O_{\text {c }}$.

In a similar way, and by collecting existing results, we can treat the case of eight dimensions. Consider Bugaenko's polytope $P_{B_{8}} \subset \mathbb{H}^{8}$ associated to the group $B_{8}$ whose Coxeter graph (with a twofold symmetry) is given in Fig. 3.

In [33, Ex. 3], Zehrt showed that the polytope $P_{B_{8}}$ has combinatorial $f$-vector

$$
(41,164,316,374,294,156,54,11)
$$

and determined the covolume of the group $B_{8}$ in terms of

$$
\chi\left(\mathbb{H}^{8} / B_{8}\right)=\frac{24,187}{8,709,120,000} .
$$

Consider the (arithmetic) $\mathbb{Z}_{2}$-extension $B_{8}^{\prime}$ of $B_{8}$. Then, the orientable double cover of $\mathbb{H}^{8} / B_{8}^{\prime}$ has Euler characteristic given by (4.6) which coincides with the minimal one for $n=8$ as given above, that is,

$$
\chi\left(\mathbb{H}^{8} / B_{8}\right)=\chi\left(O_{c}\right) .
$$

Again, by the uniqueness result of Theorem 2, we can conclude the following.

Proposition 2 The orientable double cover of the orbifold $\mathbb{H}^{8} / B_{8}^{\prime}$ is the unique orientable compact arithmetic 8-orbifold $O_{\mathrm{c}}$ of minimal volume.

\subsection{The odd-dimensional compact case}

In [7, Thm. 1.3], Emery considered orientable arithmetic hyperbolic $n$-orbifolds of minimal volume for odd $n \geq 5$. He exploited Prasad's volume formula by using techniques similar to [1,2] but he had to overcome additional obstacles arising for $\operatorname{Isom}^{+}\left(\mathbb{H}^{n}\right) \cong P O(n, 1)^{\circ}$ with $n$ odd since its algebraic simply connected covering is a 4-covering and since it is of type $D$ having outer forms. His results were made more precise in [3, Thm. 2] with respect to uniqueness and the (non-trivial) determination of certain subgroup indices. In this way, volume minimality results for orientable arithmetic orbifolds of odd dimensions $n \geq 5$ arise which, in the compact case, can be stated as follows (see also Theorem 6). 
Theorem 4 For each dimension $n=2 r-1 \geq 5$, there is a unique orientable compact arithmetic hyperbolic $n$-orbifold $O_{\mathrm{c}}$ of minimal volume. It is defined over $k_{0}=\mathbb{Q}[\sqrt{5}]$ and of volume given by the formula

$$
\operatorname{vol}_{n}\left(O_{\mathrm{c}}\right)=\frac{5^{r^{2}-r / 2} \times 11^{r-1 / 2} \times(r-1) !}{2^{2 r-1} \pi^{r}} L_{\ell_{0} \mid k_{0}}(r) \prod_{i=1}^{r-1} \frac{(2 i-1) !^{2}}{(2 \pi)^{4 i}} \zeta_{k_{0}}(2 i),
$$

where $\ell_{0}$ is the quartic field with a defining polynomial $x^{4}-x^{3}+2 x-1$.

Based on this result, we shall first present concrete results in dimension five and then discuss small volume in seven dimensions.

\subsubsection{Minimal volume realisation in dimension five}

Consider the cocompact arithmetic Coxeter prism group $\Sigma_{5}=[5,3,3,3,3]$ [see (2.2d)] with defining field $k_{0}=\mathbb{Q}[\sqrt{5}]$, and let $\ell_{0} \cong \mathbb{Q}[x] /\left(x^{4}-x^{3}+3 x-1\right)$ as in Theorem 4 . The following result has been proved in [10].

Theorem 5 The orientable double cover of the Coxeter orbifold $\mathbb{H}^{5} / \Sigma_{5}$ is the unique orientable compact arithmetic hyperbolic 5-orbifold $O_{\mathrm{c}}$ of minimal volume. Its value equals

$$
\operatorname{vol}_{5}\left(O_{c}\right)=\frac{9 \sqrt{5}^{15}}{2^{15} \pi^{15}} \zeta_{k_{0}}(2) \zeta_{k_{0}}(4) L_{\ell_{0} / k_{0}}(3) \simeq 0.00076729618 .
$$

For the proof, and inspired by the numerical coincidence of the prism volume $\operatorname{vol}_{5}\left(\Sigma_{5}\right)$ with the composed $L$-values in (4.8) [see also (2.9)], the three smallest compact arithmetic hyperbolic five-orbifolds were determined by adapting suitably the techniques developed in $[3,7]$. In this way, it was possible to identify-in a rigorous way-the rotation subgroup $\Sigma_{5}^{\prime}$ of $\Sigma_{5}=[5,3,3,3,3]$ with the covolume minimiser as stated above.

\subsubsection{About minimal volume in dimension seven}

Consider Bugaenko' s cocompact arithmetic Coxeter group $B_{7}$ with 11 generators and with Coxeter graph given by Fig. 4 . Recall that $B_{7}$ is the Coxeter group associated to the automorphism group $O\left(f_{7}, \mathbb{Z}[\omega]\right), \omega=(\sqrt{5}+1) / 2$, of the quadratic form $f_{7}$ as given by (4.3).

Again, the Coxeter graph of $B_{7}$ has an internal symmetry, and we denote by $B_{7}^{\prime}$ the $\mathbb{Z}_{2}$-extension of the group $B_{7}$, accordingly. As already mentioned in 4.1 .1 , the group $B_{7}$ is the unique Coxeter group, with compact fundamental polytope in $\mathbb{H}^{7}$, having

Fig. 4 The Bugaenko group $B_{7}$

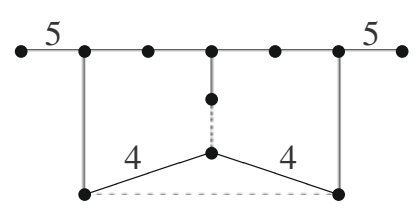


(the minimal number of) 11 natural generators. Now, all the results discussed so far indicate that minimal volume hyperbolic orbifolds are built upon (halves of) Coxeter polytopes with a minimum number of facets (and large dihedral angles), and that they are all arithmetic. However, as indicated to us by Emery [9], the orientable double cover of $\mathbb{H}^{7}$ by the group $B_{7}^{\prime}$ cannot be equal to the (unique) orientable arithmetic seven-orbifold $O_{\mathrm{c}}$. In fact, on the one hand side, the orbifold $O_{\mathrm{c}}$ stems from a certain algebraic group $G_{0}$, with splitting field $\ell_{0}$ of discriminant of absolute value 275 (see Theorem 4), and which can be related to the quadratic form

$$
x_{1}^{2}+\cdots+x_{6}^{2}-(2 \sqrt{5}-3) x_{7}^{2}
$$

On the other hand side, the "splitting field" $\ell_{1}$ of the algebraic group $G_{1}$ related to the form $f_{7}$ is the field $\mathbb{Q}[\sqrt{\omega}]$ with discriminant of absolute value 400 . Since these discriminants do not coincide, the algebraic groups $G_{0}$ and $G_{1}$ are not isomorphic which implies that the fundamental group of $O_{\mathrm{c}}$ and Bugaenko's group $B_{7}^{\prime}$ are not commensurable. Consequently, the following natural questions arise.

Question 1 Is the fundamental group of the orientable compact arithmetic hyperbolic 7-orbifold $O_{\mathrm{c}}$ of minimal volume commensurable to a hyperbolic Coxeter group at all?

Question 2 What is the precise volume, necessarily larger than $\operatorname{vol}_{7}\left(O_{c}\right)$, of the orientable double cover $O_{B_{7}^{\prime}}$ of $\mathbb{H}^{7}$ by the group $B_{7}^{\prime}$ ?

Notice that, for dimensions $n \geq 9$, we do not know about any concrete compact hyperbolic $n$-Coxeter polytope. What we do have, however, is the non-existence result of Vinberg (see [32, Part II, Cha. 5, Sec. 2.5], for example) stating that compact hyperbolic Coxeter $n$-polytopes do not exist for $n \geq 30$ !

\subsection{The odd-dimensional non-compact case}

In [7, Thm. 1.4], and with the improvement in [3, Thm. 2], Emery and EmeryBelolipetsky provide an analogous minimality result for orientable non-compact arithmetic orbifolds of odd dimensions $n \geq 5$.

Theorem 6 For each dimension $n=2 r-1 \geq 5$, there is a unique orientable noncompact arithmetic hyperbolic $n$-orbifold $O_{\mathrm{nc}}$ of minimal volume. Its volume is given by the following formula.

(1) If $r \equiv 1(\bmod 4)$ :

$$
\operatorname{vol}_{n}\left(O_{\mathrm{nc}}\right)=\frac{1}{2^{r-2}} \zeta(r) \prod_{i=1}^{r-1} \frac{(2 i-1) !}{(2 \pi)^{2 i}} \zeta(2 i)
$$

(2) If $r \equiv 3(\bmod 4)$ :

$$
\operatorname{vol}_{n}\left(O_{\mathrm{nc}}\right)=\frac{\left(2^{r}-1\right)\left(2^{r-1}-1\right)}{3 \times 2^{r-1}} \zeta(r) \prod_{i=1}^{r-1} \frac{(2 i-1) !}{(2 \pi)^{2 i}} \zeta(2 i) ;
$$




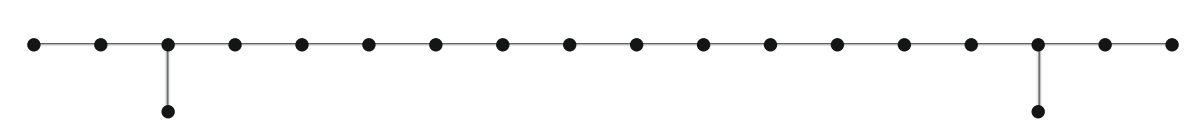

Fig. 5 The graph $\Sigma_{*}$ of the non-compact Coxeter pyramid $P_{*} \subset \mathbb{H}^{17}$

(3) If $r$ is even:

$$
\operatorname{vol}_{n}\left(O_{\mathrm{nc}}\right)=\frac{3^{r-1 / 2}}{2^{r-1}} L_{\ell_{1} \mid \mathbb{Q}}(r) \prod_{i=1}^{r-1} \frac{(2 i-1) !}{(2 \pi)^{2 i}} \zeta(2 i)
$$

where $\ell_{1}=\mathbb{Q}[\sqrt{-3}]$.

\subsubsection{The absolute minimal volume orbifold is 17-dimensional}

Consider the even unimodular lattice $\mathrm{II}_{17,1}$ in $\mathbb{R}^{18}$ equipped with the standard Lorentzian form

$$
q_{17,1}(x)=x_{1}^{2}+\cdots+x_{17}^{2}-x_{18}^{2}
$$

It is well-known that the group $P O\left(\mathrm{II}_{17,1}\right)$ of automorphisms leaving invariant $q_{17,1}$ and the lattice $\mathrm{II}_{17,1}$ is a discrete group of hyperbolic isometries. It is reflective with subgroup of index two given by the Coxeter group $\Sigma_{*}$ described by the graph in Fig. 5 . Observe that $\Sigma_{*}$ has an internal order 2 symmetry, giving rise to the $\mathbb{Z}_{2}$-extension $\Sigma_{*}^{\prime}$, as usual.

By passing to the subgroup $P S O\left(\mathrm{II}_{17,1}\right)=P O\left(\mathrm{II}_{17,1}\right) /\{ \pm I\}$ of special automorphisms, Emery proved in [8, Thm. 1] that the quotient space $\mathbb{H}^{17} / P S O\left(\mathrm{II}_{17,1}\right)$ is the orientable non-compact arithmetic hyperbolic 17-orbifold of minimal volume. As a bi-product of this result and of Theorem 6, (1), the volume of the non-compact Coxeter pyramid $P_{*} \subset \mathbb{H}^{17}$ associated to $\Sigma_{*}$ can be expressed as follows (see [8, Sec. 3]).

$$
\operatorname{vol}_{17}\left(P_{*}\right)=\frac{691 \times 2617}{2^{38} \times 3^{10} \times 5^{4} \times 7^{2} \times 11 \times 13 \times 17} \zeta(9)
$$

Furthermore, by comparing the different minimal values in even and odd dimensions $n \geq 2$ (see [1,3]), Emery's results [8] imply that the orientable double cover $O_{*}$ of the non-compact arithmetic 17 -orbifold $\mathbb{H}^{17} / \Sigma_{*}^{\prime}$ is an absolute volume minimiser in the following sense.

Theorem 7 Any orientable arithmetic hyperbolic $n$-orbifold $O$ of dimension $n \geq 2$ satisfies

$\operatorname{vol}_{n}(O) \geq \operatorname{vol}_{17}\left(O_{*}\right)=\frac{691 \times 2617}{2^{38} \times 3^{10} \times 5^{4} \times 7^{2} \times 11 \times 13 \times 17} \zeta(9) \simeq 2.072451981 \times 10^{-18}$,

with equality if and only if $O$ is isometric to $O_{*}$. 
Acknowledgments The author would like to thank Vincent Emery for helpful discussions concerning Sec. 4.2.2. and Matthieu Jacquemet for his technical support. She was partially supported by Schweizerischer Nationalfonds 200020-144438.

\section{References}

1. Belolipetsky, M.: On volumes of arithmetic quotients of $\mathrm{SO}(1, \mathrm{n})$. Ann. Sc. Norm. Super. Pisa Cl. Sci. (5) 3(4), 749-770 (2004)

2. Belolipetsky, M.: Addendum to: "On volumes of arithmetic quotients of $\mathrm{SO}(1, n)$ ". Ann. Sc. Norm. Super. Pisa Cl. Sci. (5) 6(2), 263-268 (2007)

3. Belolipetsky, M., Emery, V.: On volumes of arithmetic quotients of $\mathrm{PO}(n, 1)^{\circ}, n$ odd. Proc. Lond. Math. Soc. 105(3), 541-570 (2012)

4. Bugaenko, V.O.: Groups of automorphisms of unimodular hyperbolic quadratic forms over the ring $\mathrm{Z}[(\sqrt{5}+1) / 2]$. Mosc. Univ. Math. Bull. 5, 6-14 (1984)

5. Bugaenko, V. O.: Arithmetic crystallographic groups generated by reflections, and reflective hyperbolic lattices. In: Lie groups, their discrete subgroups, and invariant theory, Adv. Soviet Math., vol. 8, Amer. Math. Soc., Providence, RI, 1992, pp. 33-55.

6. Chinburg, T., Friedman, E.: The smallest arithmetic hyperbolic three-orbifold. Invent. Math. 86(3), 507-527 (1986)

7. Emery, V.: Du volume des quotients arithmétiques de l'espace hyperbolique. PhD thesis, University of Fribourg (2009)

8. Emery, V.: Even unimodular lorentzian lattices and hyperbolic volume. J. Reine Angew. Math. 2014(690), 173-177 (2012)

9. Emery, V.: Private communication (2013)

10. Emery, V., Kellerhals, R.: The three smallest compact arithmetic hyperbolic 5-orbifolds. Algebr. Geom. Topol. 13, 817-829 (2013)

11. Felikson, A., Tumarkin, P., Zehrt, T.: On hyperbolic Coxeter $n$-polytopes with $n+2$ facets. Adv. Geom. 7(2), 177-189 (2007)

12. Gehring, F.W., Maclachlan, C., Martin, G.J., Reid, A.W.: Arithmeticity, discreteness and volume. Trans. Am. Math. Soc. 349(9), 3611-3643 (1997)

13. Gehring, F.W., Martin, G.J.: Precisely invariant collars and the volume of hyperbolic 3-folds. J. Differ. Geom. 49(3), 411-435 (1998)

14. Gehring, F.W., Martin, G.J.: Minimal co-volume hyperbolic lattices, I: the spherical points of a Kleinian group. Ann. Math. 170(1), 123-161 (2009)

15. Grunewald, F., Kühnlein, S.: On the proof of Humbert's volume formula. Manuscr. Math. 95(4), 431436 (1998)

16. Hild, T.: The cusped hyperbolic orbifolds of minimal volume in dimensions less than ten. J. Algebra 313(1), 208-222 (2007)

17. Hild, T., Kellerhals, R.: The fcc lattice and the cusped hyperbolic 4-orbifold of minimal volume. J. Lond. Math. Soc. 75, 677-689 (2007)

18. Johnson, N.W., Ratcliffe, J.G., Kellerhals, R., Tschantz, S.T.: The size of a hyperbolic Coxeter simplex. Transform. Groups 4(4), 329-353 (1999)

19. Kellerhals, R.: On the volume of hyperbolic polyhedra. Math. Ann. 285(4), 541-569 (1989)

20. Kellerhals, R.: On Schläfli's reduction formula. Math. Z. 206(2), 193-210 (1991)

21. Kellerhals, R.: Volumes of cusped hyperbolic manifolds. Topology 37(4), 719-734 (1998)

22. Kellerhals, R.: Scissors congruence, the golden ratio and volumes in hyperbolic 5-space. Discret. Comput. Geom. 47(3), 629-658 (2012)

23. Kellerhals, R., Perren, G.: On the growth of cocompact hyperbolic Coxeter groups. Eur. J. Combin. 32(8), 1299-1316 (2011)

24. Marshall, T.H., Martin, G.J.: Minimal co-volume hyperbolic lattices, II: simple torsion in a Kleinian group. Ann. Math. (2) 176(1), 261-301 (2012)

25. Meyerhoff, R.: The cusped hyperbolic 3-orbifold of minimum volume. Bull. Am. Math. Soc. (N.S.) 13(2), 154-156 (1985)

26. Milnor, J.: Hyperbolic geometry: the first 150 years. Bull. Am. Math. Soc. (N.S.) 6(1), 9-24 (1982)

27. Siegel, C.L.: Some remarks on discontinuous groups. Ann. Math. 46(4), 708-718 (1945) 
28. Tumarkin, P.: Hyperbolic Coxeter polytopes in $\mathbb{H}^{m}$ with $n+2$ hyperfacets. Mat. Zametki $75(6)$, 909-916 (2004)

29. Tumarkin, P.: Compact hyperbolic Coxeter n-polytopes with $n+3$ facets. Electron. J. Combin. 14(1) (2007). Research Paper 69 (electronic)

30. Tumarkin, P., Felikson, A.: On bounded hyperbolic d-dimensional Coxeter polytopes with $d+4$ hyperfaces. Tr. Mosc. Mat. Obs. 69, 126-181 (2008)

31. Vinberg, E.B.: Hyperbolic groups of reflections. Uspekhi Mat. Nauk. 40, 241(1), 29-66, 255 (1985)

32. Vinberg, È.B., Shvartsman, O.V.: Discrete groups of motions of spaces of constant curvature. In: Geometry, II, Encyclopedia Math. Sci., vol. 29, pp. 139-248. Springer, Berlin (1993)

33. Zehrt, T.: The covolume of discrete subgroups of Iso $\left(\mathbb{H}^{2 m}\right)$. Discret. Math. 309(8), 2284-2291 (2009) 